\title{
The effect of spatial separation of response and reinforcement on DRL performance'
}

CHARLES D. CORMAN 2 AND JAMES N. SHAFER WEST VIRGINIA UNIVERSITY

The distribution of IRT's between 0 and $3 \mathrm{sec}$. and collateral behavior in a DRL schedule were studied with the bar spatially separated from the reinforcement. The distribution of very short IRT's for the four rats stabilized after $25 \mathrm{hr}$. of training. This suggests that stimuli involved in consummatory activity do not adventitiously reinforce "bursts" of responses. The animal's rate of locomotion to and from the food tray adjusted to the interval between reinforcements.

It is well established that a DRL schedule, in general, produces a characteristic distribution of inter-response times (IRTs). Different experimenters, however, have reported differing relative frequencies of very short IRTs, i.e., IRTs which are classified in the smallest class interval. Sidman $(1955,1956 \mathrm{a}, 1956 \mathrm{~b})$, using liquid reinforcement, reported a high proportion of very short IRTs which was stable with prolonged training. Kelleher et al (1959), using food pellets as reinforcers and failing to find a similar high proportion of very short IRTs suggested that the discrepancy was "undoubtedly caused by differences in deprivation conditions, reinforcers, or apparatus" (p. 105). They pointed out, by way of example, that the investigators reporting a high proportion of very short IRTs were those who consistently used liquid reinforcers delivered by a motor-driven cup and suggested that a second response occurring during the brief delay in delivery by the mechanism might be adventitiously reinforced. Further investigation, however, has shown that the use of liquid reinforcement does not guarantee very short IRTs (Laties \& Weiss, 1962). Since the proportion of very short IRTs appears independent of the reinforcing agent, the effects of other factors must be assessed.

In the standard operant test apparatus, the food tray or water delivery cup) is adjacent to the operandum making it possible for the animal to respond while ingesting the reinforcing agent, thus reinforcing additional responses and producing very short IRTs. The present experiment was performed to assess changes in the IRT distribution as a function of displacing the food cup from its usual position adjacent to the bar to a position in the rear of the test chamber. This change in apparatus, by spatially, and thus temporally separating the reinforcement from the response, will prevent reinforcement of very short IRTs by the delay in ingestion of the food pellets. In addition, by separating the food tray from the operandum and thus extending the range of behavior the animal engages in, it should be possible to observe whether stereotyped chains of collateral behavior develop as found by some investigators (Wilson \& Keller, 1953; Bruner \& Revusky, 1961; Hodos et al, 1962; Laties et al, 1965) but not by others (Anger, 1956; Kelleher et al, 1959).

Method

Four male, experimentally naive, hooded rats were used as Ss. The animals were from the same litter, 72 days old at the beginning of the experiment. The animals were designated $D-1, D-2, D-3$, and $D-4$.

The apparatus consisted of a small animal test chamber equipped with a bar and automatic pellet dispenser. The food cup was at the rear of the compartment, 8 in. from the bar. Responses and reinforcements were recorded by electrical impulse counters which tabulated IRTs ranging from 0 to $27 \mathrm{sec}$. in class intervals of $3 \mathrm{sec}$. The test chamber was inside a sound shielded room with the programming and analyzing equipment in an adjacent room. $A$ window and mirror arrangement permitted observation of the animals' behavior.

The animals were placed on a 22-hr. food deprivation schedule five days prior to the beginning of pretraining and adapted to the apparatus. Bar pressing was then established by giving each animal a total of 100 continuous reinforcements over a ten-day period. The reinforcement schedule was then changed to DRL$15 \mathrm{LH}-12$, and training sessions initiated. Experimental sessions were of $1-\mathrm{hr}$. duration Monday through Friday and continued for $45 \mathrm{hr}$.

\section{Results and Discussion}

The response rates for the four Ss during exposure to the schedule are shown in Fig. 1. After approximately $10 \mathrm{hr} .$, a stable rate was established which was maintained throughout testing. The similarity of the

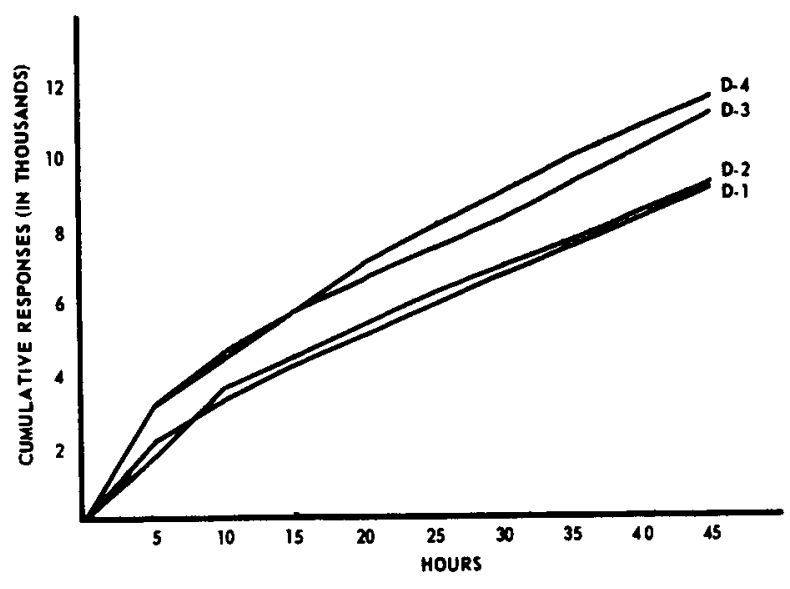

Fig. 1. Cumulative Responses During Testing. 


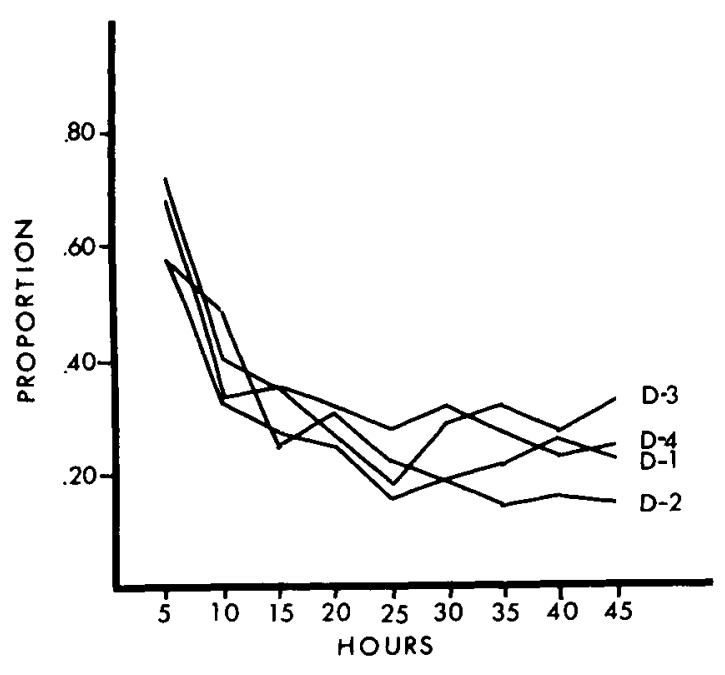

Fig, 2. Proportion of 0-3 sec. IRTs.

slopes suggests that a given rate of responding is associated with the experimental situation.

The proportion of very short IRTs obtained in the experiment are presented in Fig. 2. Each value represents the relative frequency of 0-3 sec. IRTs for a 5-hr. block of sessions. It would appear that the proportion of very short IRTs stabilized after approximately $25 \mathrm{hr}$., and at values close to those obtained by Sidman. The results of the experiment, therefore, indicate that the stimuli involved in consummatory behavior do not reinforce responses producing very short IRTs.

Observation of the animals' behavior revealed a stereotyped temporal pattern of locomotion. Upon the operation of the pellet dispenser the S would typically turn and approach the food cup. As training continued, the animal slowed his locomotion to and from the food cup to a point where the time between bar presses specified by the DRL schedule was consumed. If the bar press were unreinforced, the animal would remain active, moving about the chamber or licking and scratching at the food cup in no consistent pattern.
In an earlier study (Corman \& Shafer, 1962) using a standard operant chamber with the food cup adjacent to the bar, it was observed that animals showed stereotyped inter-response behaviors during the time when the characteristic DRL temporal distribution of responses was emerging, but that with extended training these chains of behavior disappeared. It was suggested that these chains served to mediate the formation of a temporal discrimination based on internal cues. In the present study, changes in the rate of locomotion may have assumed the function of such chains.

\section{References}

Anger, D. The dependence of interresponse times upon the reliztive reinforcement of different interresponse times. $J$. exp. Psychol., 1956, 52, 145-161.

Buner, A., \& Revusky, S. H. Collateral behavior in humans. $J$. exp. Anal. Behav., 1961, 4, 349-350.

Corman, C. D., \& Shafer, J. N. The effect of patterns of background stimuli on the timing behavior of the rat. Proc. W. Va. Acad. Sci., 1962, 34, 183-188.

Hodos, W. G., Ross, S., \& Brady, J. V. Complex response patterns during temporally spaced responding. J. exp. Anal. Behav., $1962,5,473-479$.

Kelleher, R. T., Fry, W., \& Cook, L. Interresponse time distribution as a function of differential reinforcement of temporally spaced responses. J. exp. Anal. Behav., 1959, 2, 91-106.

Laties, V. G., \& Weiss, B. Effects of alcohol on timing behavior. J. comp. physiol. Psychol., 1962, 55, 85-91.

Laties, V. G., Weiss, B., Clark, L., \& Reynolds, M. D. Overt "mediating" behavior during temporally spaced responding. $J$. exp. Anal. Behav., 1965, 8, 107-116.

Sidman, M. Technique for the assessment of drug effects on timing behavior. Science, 1955, 122, 925.

Sidman, M. Time discrimination and behavioral interaction in a free operant situation. J. comp. physiol. Psychol., 1956a, 49, 469-473.

Sidman, M. Drug-behavior interaction. Ann. N. Y. Acad. Sci., $1965 a, 65,282-302$

Wilson, M. P., \& Keller, F. S. On the selective reinforcement of spaced responses. J. comp. physiol. Psychol., 1953, 46, 190193.

\section{Notes}

1. Based on a Master's thesis by the first author under the direction of the second.

2. Now at the University of Southern California. 